\title{
Влияние неопределенности фондового рынка на инвестиционную активность российских компаний через формирование цен на базовые активы
}

\author{
Черкасова В.А. ${ }^{14}$, Мочалов Д.С. ${ }^{15}$
}

В данной статье исследуется влияние неопределенности, возникающей на срочном рынке при формировании цен на производные финансовые инструменты, базовым активом для которых является основная продукция крупных российских добывающих компаний. Цены, устанавливающиеся на производные финансовые инструменты, являются ожиданиями относительно будущих изен на базовые активы срочных инструментов. Такие данные являются неким агрегированным прогнозом будущей ситуации, которую компании должны принимать во внимание при формировании своей инвестиционной политики.

JEL: G31, G32, G11

Ключевые слова: инвестиционная активность, капитальные вложения, неопределенность фондового рынка, базовые активы, срочный рынок

Рынок производных ценных бумаг может служить инструментом прогнозирования для компаний, что позволит последним сделать процессы планирования, в том числе инвестиционного, более простыми и прозрачными.

На срочном рынке (рынке производных финансовых инструментов) на различные товары устанавливаются контракты (фьючерсы и опционы) со сроками исполнения, кратными кварталу, такие контракты могут заключаться на несколько лет вперед. Устанавливающиеся на этом рынке цены на соответствующие контракты можно назвать рыночным прогнозом стоимости актива на конкретную дату в будущем. Если считать, что рынок совершенен и доступна вся информация, то никакой финансовый инструмент не может стоить дороже, чем будет его реальная стоимость. При таких условиях цена контракта должна равняться разнице между прогнозируемой участником торгов ценой товара в будущем и его стоимостью, прописанной в контракте.

Существуют два вида ценных бумаг, базовыми активами для которых являются товары. По одним контрактам предусмотрены реальные поставки товаров, когда при исполнении одна из сторон обязана осуществить реальную поставку обозначенного в контракте товара по цене. При этом в случае опционов сторона, владеющая им, может не исполнять свои обязательства, выплатив в итоге лишь саму стоимость опциона. Данный вид контрактов может использоваться компаниями для хеджирования рисков, связанных с обеспечением бесперебойных поставок клиентам, однако он может применяться только компаниями из соответствующей отрасли, занимающимися продажами таких товаров, а также потребителями крупных партий товаров.

Второй вид производных ценных бумаг, базовыми активами для которых являются товары, не предполагает реального обеспечения поставок самих товаров. Такие контракты заключаются на стоимость товаров, которая, как правило, берется с таких рынков, как Platts, на которых формируются индексы цен на товары, а также активно торгуются срочные контракты на товары, предусматривающие фактическую поставку. Такими инструментами могут пользоваться не только компании, которые работают с товарами, являющимися базовыми активами для производных ценных бумаг, но и другие участники фондового рынка (в России к торгам такими инструментами допускаются только квалифицированные

\footnotetext{
${ }^{14}$ Канд. эконом. наук, доцент кафедры экономики и финансов фирмы НИУ ВШЭ.

${ }^{15}$ Магистр экономики, главный экономист / инвестиционный аналитик ООО «Газ-ойл».
} 
инвесторы). Тем не менее такие ценные бумаги полностью отражают все тенденции изменения цен как самих базовых активов, так и контрактов, предполагающих реальную поставку товаров.

Цены на оба вида контрактов формируются из двух составляющих: одной из них является текущая рыночная цена на товар (как правило, на зарубежных товарных биржах котировки доступны за каждый день по тем же товарам, на которые установлены производные ценные бумаги); а второй составляющей являются ожидания относительно будущих цен на тот же товар. Несмотря на сложность расчетов стоимости производных бумаг следует ожидать, что влияние неопределенности относительно цен таких активов на инвестиционную активность компаний, так же как и влияние неопределенности относительно других параметров, окажется отрицательной.

Исследования влияния различных видов неопределенности на инвестиционную активность компаний занимают значительное место в современной литературе. Есть два больших класса работ по данной тематике: одни исследуют влияние шоков спроса и предложения и вызванной их существованием неопределенности (Abel, Eberly, 1997), другие посвящены влиянию неопределенности фондового рынка на инвестиционную активность компаний (Branston, Groenewold, 2004). Работы, исследующие влияние фондового рынка, традиционно рассматривают колебания на рынке акций компаний, так как при использовании предпосылки о совершенной информации на рынке в ценах акций должна корректно отражаться вся доступная информация о компании. Акции являются одним из источников средств для компании, а также их стоимость влияет на стоимость капитала компании. Акции могут использоваться в качестве залога или обеспечения по кредитам, и также при оценке кредиторами рисков учитывается устойчивость и динамика цен акций компаний на рынке. Все эти факторы обеспечили пристальное внимание именно к акциям, и, несмотря на то что целью данной статьи является исследование влияния неопределенности рынка производных финансовых инструментов, методология, разработанная для акций, может лечь в основу методологии, разрабатываемой в данной статье.

Существует несколько теорий о том, каким именно образом фондовый рынок и возникающая на нем неопределенность влияют на инвестиционную активность компаний. Некоторые из них разработаны специально под рынок акций и не могут быть применены при исследовании влияния других сегментов рынка, однако есть и такие, которые могут быть адаптированы и применены к рынку производных финансовых инструментов. Одно из базовых исследований в данной области, проведенное Морком (Morck et al., 1990), описывает четыре теории о влиянии фондового рынка на инвестирование.

Теория пассивной информированности утверждает, что стоимость финансовых инструментов отражает только результаты реализованных проектов, то есть не дает менеджерам никакой новой информации о поведении инвесторов. Согласно данной теории, менеджеры при принятии решений об инвестировании не принимают в расчет мнение и оценки рынка. В этом случае любые изменения на фондовом рынке не повлияют на инвестирование в силу того, что в рамках теории стоимость акций не отражает прогноз изменения фундаментальных факторов, а отражает лишь известные события. Если интерпретировать данную теорию в приложении к рынку производных финансовых инструментов, то изменение их стоимости никак не должно повлиять на инвестиционную политику компаний. Можно предположить, что ожидания инвесторов формируются лишь исходя из прошлой и текущей стоимости базовых активов, при этом стоимость производных финансовых инструментов отражает лишь общие исторические тенденции или подчиняется закону случайного блуждания. В таком случае менеджеры не будут и не должны при принятии инвестиционных решений принимать во внимание изменение цен на производные ценные бумаги, базой для которых является производимый компанией товар.

Однако довольно сложно представить выполнение одного из основных постулатов данной теории о том, что внешний инвестор знает значительно меньше инсайдера и собирает информацию с целью понять мотивы действий менеджмента компании. Именно то, что на 
основании доступной информации происходят попытки интерпретировать действия менеджеров компании, приводит к явлению, когда может наблюдаться корреляция между инвестициями компаний и колебаниями на рынке. При проведении анализа внешние инвесторы обладают значительно меньшим количеством факторов и данных и не всегда могут абсолютно верно интерпретировать происходящее в компании, к тому же важная информация о компании гораздо раньше доходит до менеджеров. На рынке производных финансовых инструментов информация достигает инсайдеров и внешних пользователей информации одновременно; кроме того, на рынок действует не только прогнозирование по историческим тенденциям, на срочном рынке всегда присутствует и прогнозирование будущих значений факторов. Поэтому подобная теория, скорее всего, не будет иметь место при описании возможного влияния срочного рынка на инвестиционную активность компаний.

В отличие от теории пассивной информированности, теория активной информированности, напротив, предполагает, что фондовый рынок является источником информации, на основании которой строится стратегия компании (Barro, 1990). При этом информация, источником которой служит фондовый рынок, всегда включает в себя часть, основанную на фундаментальных факторах, и часть, основанную на ожиданиях инвесторов, которые зачастую бывают ошибочными. Менеджерам компаний бывает сложно отличить изменение цен, вызванное фундаментальными факторами, от изменения цен, вызванного ожиданиями инвесторов, что приводит к необходимости учитывать общее изменение цен при принятии решений. Несмотря на то что данные фондового рынка могут предоставлять менеджерам полезную информацию, которая влияет на принятие инвестиционных решений и данный источник может быть наиболее точным, подобная информация может содержать ошибку просто в силу заложенного в фундаментальные факторы элемента неопределенности. Описанные выше факторы приводят к тому, что рынок, с одной стороны, должен корректно отражать влияние фундаментальных факторов, а значит, цены содержат полезную информацию; а с другой стороны, наличие ошибок и нефундаментальных факторов приводит к существованию отклонений от равновесных значений, что также отражается на принятии инвестиционных решений.

Согласно данной теории, получается, что в случае, если рынок правильно отражает фундаментальные факторы, он не вносит ничего нового и, следовательно, для менеджеров равноценным является учет всех фундаментальных факторов самостоятельно внутри компании и учет рыночной информации. В том случае, если на показатели рынка влияют не только фундаментальные факторы, но и ожидания его участников, после корректного расчета влияния всех фундаментальных факторов инвестиционные процессы компании будут подвержены влиянию рынка (если менеджеры продолжают доверять такой информации).

Применительно к данному исследованию данная теория выглядит наиболее подходящей и жизнеспособной. С одной стороны, менеджеры компаний могут пользоваться информацией данного сегмента фондового рынка, полагая, что в цене корректно отображены фундаментальные факторы. В идеальной ситуации анализ данных такого рынка и анализ будущих значений фундаментальных факторов даст одинаковые результаты, следовательно, рынок не несет никакой дополнительной информации, которая не содержалась бы в фундаментальных факторах, а значит, на основании таких сведений можно строить инвестиционную политику компании. В то же время на срочном рынке в ценах инструментов отражаются ожидания инвесторов относительно будущей стоимости базовых активов, что вносит элемент неопределенности (Haruna, 1996). При этом отделить ожидания инвесторов достаточно трудно, вследствие чего на принятие инвестиционных решений менеджментом будет влиять общее изменение цен на производные финансовые инструменты.

Третья, так называемая финансовая теория влияния неопределенности на инвестирование заключается в том, что чем выше стоимость акций, тем дешевле становится финансирование за счет них. Данная теория не может быть применена в данной статье, так 
как производные финансовые инструменты не могут рассматриваться в качестве источников финансирования. Можно предположить, что активное использование рынка производных финансовых инструментов для хеджирования рисков могло бы положительно сказываться на инвестиционной активности компаний за счет минимизации потерь. Однако для проведения подобного рода исследований необходимо знать, насколько активно каждая компания работает на срочном рынке. К тому же крупные компании в России предпочитают не заниматься подобной деятельностью, так как для качественного осуществления хеджирования необходимо создавать специальный отдел. А в компаниях, которые все же используют такие инструменты для хеджирования, подобные операции часто приносят убытки.

Четвертая теория заключается в давлении фондового рынка на компанию. В случае если инвесторы начнут продавать ценные бумаги, то компания автоматически окажется в ситуации, когда ей придется инвестировать не в развитие своего основного бизнеса, а, например, диверсифицировать свой бизнес с целью повысить стоимость своих акций. В целях данного исследования данная теория в чистом виде не может быть применена. Однако в ситуации, когда продукция компании сильно дифференцирована и один или несколько товаров являются базой для производных ценных бумаг, падение цен на такие финансовые инструменты может привести к оттоку инвестиций от направления деятельности компании, связанного с производством базовых активов. При этом общий объем инвестиций компании может как сократиться, так и остаться неизменным. Во втором случае внутри компании произойдет перераспределение денежных инвестиционных потоков между производством разных видов продукции.

Традиционно инвестиционную активность компаний задают через размер инвестиций, или показатель Cарех в отчетности компании. Для проведения регрессионного анализа исследователи используют два типа зависимой переменной. В одних работах используется ненормированный показатель инвестиционных расходов (Bond, Van Reenen, 2003), что позволяет проводить достаточно простые в плане расчетов исследования, демонстрирующие влияние различных факторов на инвестиционную активность. Другие авторы - Брэнстон и Грёневольд (Branston, Groenewold, 2004) - используют в качестве зависимой переменной изменение логарифма инвестиций, в прямой форме выявляя, на сколько процентов изменятся инвестиции при изменении факторов. В работах Абдул-Хакю, В. Шаопинга (Abdul-Haque, Shaoping, 2008) используется нормированный показатель инвестиций на капитал, что позволяет, во-первых, применять более сложные и точные методы исследований, а вовторых, такие работы позволяют применять и тестировать влияние показателя инвестиционных возможностей Q Тобина.

Для проведения исследования в данной статье будет построена модель, зависимой переменной в которой является нормированный показатель инвестиций.

Одной из главных проблем исследования является расчет и определение фактора волатильности. В качестве прокси-переменной для фактора неопределенности чаще всего используют два схожих параметра: среднеквадратичное отклонение или стандартное отклонение.

Преимущество использования стандартного отклонения заключается в самом способе его расчета, так как оно является несмещенной оценкой, в отличие от среднеквадратичного отклонения, расчет которого в условиях, когда известны данные лишь по определенной выборке, дает смещенные результаты.

В модель для проверки гипотезы о квадратичной зависимости инвестиций и неопределенности включен показатель неопределенности (стандартные отклонения) в квадрате. Значимость данного фактора будет означать наличие квадратичной зависимости, что обусловлено тем, что компания не может откладывать инвестиции бесконечно долго, в силу чего даже при значительной неопределенности показатель инвестиционных вложений на определенном этапе должен начать расти.

Среди других переменных будет проведена проверка лаговых переменных. 
Значительная часть инвестиций производственных компаний направляется в капитальные активы, имеющие длительный срок эксплуатации, которые изнашиваются на протяжении этого срока в течение каждого периода на некоторую величину $s_{t, \tau} I_{t-\tau}$ (Сошникова, 2009). Сумма таких величин по времени составляет стоимость активов, что является сутью расчета их амортизации. Согласно модели инвестиционного акселератора (Берндт, 2005), инвестиции рассчитываются как приращение стоимости капитала с учетом его выбытия, что показывает следующее уравнение:

$$
I_{t}=K_{t}-K_{t-1}=k\left(Y_{t}-Y_{t-1}\right)=k \lambda Y_{t}-(1-\delta) k \lambda Y_{t-1}+(1-\lambda) I_{t-1} .
$$

Так как скорость подстройки капитала к его оптимальному уровню $(\lambda)$ лежит в пределах от нуля до единицы, то на теоретическом уровне получается положительное влияние лагов инвестиций на инвестиции в настоящем. Большинство авторов, исследовавших данный вопрос, приходят к однозначному выводу о положительном влиянии лаговых значений инвестиций на показатель инвестиционной активности: например, Садорски и Абель, Эберли (Sadorsky, 2008; Abel, Eberly, 1998).

Для того чтобы регрессия была доопределена, необходимо использовать такие внутренние показатели компании, как выручка и денежные потоки. Данные показатели всегда положительно влияют на инвестиционную активность компаний в силу того, что с их ростом увеличиваются внутренние источники для финансирования компании. В модель включены лаговые показатели денежного потока и выручки, которые могут оказывать значимое влияние на инвестиции компании (Drakos, Goulas, 2006).

Вышеперечисленные факторы являются основными, но не исчерпывают возможности модели. Так, для проверки результатов в регрессию может быть добавлена волатильность базовых активов. Данный фактор не может быть напрямую включен в регрессию вместе с фактором неопределенности, который задается как стандартное отклонение цен производных инструментов, последний зависит от волатильности цен на базовые активы. Возникающая при этом мультиколлинеарность может быть крайне сильной, дестабилизируя результаты исследования.

Некоторые другие факторы, также влияющие на инвестиционную активность компаний, такие как структура капитала или уровень долга компании, в данной статье не отражены, для того чтобы регрессия не получилась переопределенной.

Таким образом, предполагается проверить в модели гипотезы об отрицательном влиянии неопределенности и лаговых показателей инвестиционной активности, а также о положительном влиянии выручки и денежных потоков на инвестиционную активность.

Исследование проводится по следующему алгоритму:

- На первом этапе подбираются факторы, которые могут оказывать значимое влияние на инвестиционную активность компаний. Формируется регрессионное уравнение.

- На втором этапе происходит выбор наилучшей модели между моделями с фиксированными и случайными эффектами и сквозной регрессией.

- На третьем этапе исследуется зависимость фактора неопределенности, возникающей на срочном рынке, от прочих факторов.

- На четвертом этапе проводится тестирование регрессионного уравнения, построенного с учетом значимых факторов, определенных на третьем этапе.

- На пятом этапе проводится исследование колебаний фактора неопределенности во времени. Методом имитационного моделирования проводится формирование полиномиальной регрессии, позволяющей делать выводы о периодах наибольшей волатильности цен финансовых инструментов.

\section{Формирование выборки и описание данных}

Исследование проводится на выборке, включающей в себя данные по 49 российским нефтегазовым, золотодобывающим, добывающим медь, серебро и палладий компаниям. В 
выборку вошли и компании, тесно связанные с добывающими компаниями, то есть такие, чьи доходы косвенно зависят от ситуации на рынках базовых активов. Например, доход компании «Транснефть» фактически зависит от стоимости нефти, которую она транспортирует.

Данные по компаниям собирались в базах Bloomberg и Ruslana, представленной Bureau Van Dijk, а также были взяты из отчетности компаний. Рассматривался период с конца 2008 по 2011 год в поквартальной разбивке.

Данные были подобраны по таким показателям, как Сарех (объем инвестиционных затрат), выручка, активы (показатель Total Assets), денежные потоки. Дополнительно были подобраны показатели Q Тобина, рыночной стоимости компаний и суммарного долга.

Основная модель выглядит следующим образом:

$I N V=\alpha+\beta_{1} I N V \_L A G 1+\beta_{2} U N C+\beta_{3} U N C \_S Q+\beta_{4} S L S+\beta_{5} S L S \_L A G 1+\beta_{6} C F+$

$+\beta_{7} C F \_L A G 1+\bar{\varepsilon}$,

где:

INV - нормированный на капитал показатель инвестиций; рассчитывается как Capex/Total Assets, где Capex есть показатель инвестиций в период $t$, а Total Assets есть размер активов компании согласно отчетности на конец предыдущего периода;

$I N V \_L A G 1$ - лаговый показатель инвестиций; рассчитывается аналогично показателю $I N V$;

$U N C$ - показатель неопределенности, рассчитываемый как стандартное отклонение стоимости финансовых инструментов;

$U N C \_S Q$ - показатель неопределенности в квадрате;

$S L S$ - нормированный на капитал показатель выручки;

$S L S \_L A G 1$ - лаговый показатель выручки (нормированный);

$C F$ - нормированный на капитал показатель денежного потока компании;

$C F \_L A G 1$ - лаговый показатель денежного потока;

$\varepsilon_{i t}$ - случайная ошибка, распределенная по нормальному закону.

Модель сформулирована и построена таким образом для того, чтобы можно было ввести в качестве проверочного показателя фактор Q Тобина. Ожидается, что в модели такого вида фактор окажется незначимым или слабозначимым и не будет иметь большой объясняющей силы.

Для оценки влияния неопределенности на рынке производных финансовых инструментов, а также других факторов на инвестиционную активность компании для каждой спецификации модели оценивались три вида регрессий: сквозные регрессии, регрессии с наличием индивидуального фиксированного эффекта (Fixed effect), а также регрессии с наличием случайного эффекта (Random effect). Оценка проводилась с применением пакета статистического анализа EViews.

Выбор между моделями с фиксированным и случайным эффектами проводился с помощью теста Хаусмана. Выбор между моделью с фиксированными эффектами и сквозной регрессией осуществлялся с помощью проведения F-теста.

\section{Определение значимости и влияния факторов на инвестиционную активность компаний}

На первом этапе исследования проводилась оценка модели с фиксированным эффектом. По итогам проведенных тестов была выбрана именно эта модель, в то время как модель со случайными эффектами и сквозная регрессия показали худшие результаты.

После того как предпочтение будет отдано модели с фиксированными эффектами, необходимо задать ее правильную спецификацию, так как в модели присутствуют незначимые переменные.

Как следует из анализа модели с фиксированными эффектами, в ней одна незначимая переменная, а именно лаговый показатель выручки (для $t$-статистики $p$-value составляет 
0,7533). Качество модели возросло после того, как из нее была ликвидирована незначимая переменная. Нормированный $R$-квадрат вырос до 0,6273. Значение коэффициента детерминации составляет 0,7049 , что является признаком качества регрессии. При этом незначимым на 10\%-ном уровне становится показатель квадратичной меры неопределенности ( -value 0,1208), что заставляет рассмотреть возможность ликвидации показателя из регрессии. При попытке убрать данный показатель наблюдалось падение нормированного и простого показателей $R$-квадрат, что свидетельствует о снижении качества регрессии. Было решено оставить данный показатель в модели. В итоговой модели все переменные значимы.

Данная аналитика показывает, что регрессия значима уже на 1\%-ном уровне значимости $(p$-value $=0,000)$. Для того чтобы полученные в модели оценки коэффициентов были состоятельными и эффективными, важно отсутствие в модели таких проблем, как гетероскедастичность, мультиколлинеарность и автокорреляция. Для того чтобы избежать проблемы гетероскедастичности, в пакете Stata строилась модель, очищенная от нее. Автокорреляция в модели также отсутствует, что отражено в показателе Durbin-Watson stat $(D W$ stat $=1.705)$. Можно сделать вывод об отсутствии мультиколлинеарности, так как корреляция показателей не превышает $26 \%$.

Итоговая модель имеет следующий вид:

$I N V=-0,254166+0,053663 * I N V \_L A G 1-0,213889 * U N C+0,002553 * U N C \_S Q++$ $0,246911 * S L S+0,087586 * C F+0,030857^{*} C F \_L A G 1$,

Влияние показателей, полученных в данной регрессии, соответствует основным теориям, изложенным выше. Стоит отметить, что по результатам модели можно увидеть сильное влияние рыночной неопределенности, вызванной волатильностью цен на срочном рынке, на инвестиционную активность компаний.

Для проверки в данную модель был добавлен показатель Q Тобина, однако он не оказал сколько-нибудь значимого влияния на остальные факторы, тогда как остался незначим сам, что совпадает с результатами Абдул-Хакю, В. Шаопинга (Abdul-Haque, Shaoping, 2008). Значение $p$-value для показателя Q Тобина составило 0,1625 , a $R^{2}$-adjusted упал до 0,6278 , что говорит о том, что данный показатель не придает модели дополнительной объясняющей силы. Данный результат показывает, что модель подобрана правильно и включение Q Тобина, обладающего сильным эффектом в силу построения, не дестабилизировало модель.

\section{Определение факторов, влияющих на волатильность стоимости производных финансовых инструментов}

В оцениваемую модель включен фактор неопределенности, задающийся как волатильность цен производных финансовых инструментов. Стоимость производного финансового инструмента явным образом зависит от стоимости актива, который является для данного инструмента базой. Это происходит в силу следующей связи: стоимость срочного производного финансового инструмента (например, фьючерса), отражает ожидания участников рынка относительно будущих цен на базовый актив. В то же время ожидания в значительной степени базируются на текущей рыночной ситуации, а также на анализе фундаментальных факторов. Однако если говорить про цены на реальные активы, то прогнозировать их динамику, оценивая факторы, которые можно признать фундаментальными, - достаточно трудная задача. Это приводит к тому, что при определении цены производных ценных бумаг именно стоимость базового актива играет основную роль.

Если неопределенность, вызванная волатильностью цен на производные ценные бумаги, влияет на инвестиционную активность компаний, то и волатильность цен на базовые продукты также будет влиять на инвестиции компании. Однако использовать данные показатели в одной регрессии нельзя, так как в таком случае будет возникать сильная мультиколлинеарность. Поскольку такую регрессию оценивать нельзя, то целесообразно оценить, каким образом волатильность цен реальных активов влияет на волатильность цен 
производных инструментов. Для этого была построена следующая модель:

$U N C 1=\alpha+\beta_{1} U N C \_B A 1+\beta_{2}$ TIME $+\varepsilon$.

Регрессионный анализ, проведенный на примере данных для нефти сорта Brent в качестве базового актива, показал, что волатильность цен на производные финансовые инструменты значительно зависит от волатильности цен на свои базовые активы. Между тем волатильность цен производных финансовых инструментов не зависит от того, сколько месяцев до исполнения контракта осталось. Таким образом, можно утверждать, что волатильность цен базовых активов является определяющим фактором волатильности цен для производных финансовых инструментов на эти активы. В целом построенная регрессия получилась значимой ( $p$-value $<0,0000)$.

Итоговая регрессия выглядит следующим образом:

$U N C 1=5,709437+0,868699 U N C \_B A 1$.

Данная аналитика показывает, что регрессия значима уже на $1 \%$-ном уровне значимости ( $p$-value $=0,000)$, коэффициент детерминации составляет более $90 \%$.

После того как были выявлены переменные, влияющие на неопределенность, вызванную волатильностью цен производных финансовых инструментов, можно подставить данный результат в исследуемую регрессию.

Полученная после подстановки модель имеет следующий вид:

$I N V=\alpha+\beta_{1} I N V_{-} L A G 1+\beta_{2} U N C_{-} B A+\beta_{3} U N C_{-} B A S Q+\beta_{4} S L S+\beta_{5} S L S \_L A G 1+\beta_{6} C F+$

$+\beta_{7} C F \_L A G 1+\varepsilon$.

Учитывая, что сама модель не изменилась, а лишь была заменена переменная, при исследовании применялась только регрессия с фиксированным эффектом, так как было показано, что именно данный тип регрессии является предпочтительной.

В итоге значимость основных переменных, которые были использованы ранее, практически не изменилась. Лаговая переменная инвестиций, как и в ранее описанной регрессии, положительно влияет на инвестиционную активность компании, что полностью согласуется с теорией акселератора. Лаговая переменная выручки при этом остается незначимой, что идет вразрез с большинством исследований. Это можно объяснить тем, что при планировании инвестиций на год в компаниях зачастую отталкиваются от плановой выручки, а в компаниях таких отраслей, как нефть и металлургия, необходимо делать постоянные инвестиции для поддержания объемов добычи, независимо от выручки прошлых периодов.

Факторы выручки текущего года, денежных потоков и лаговых денежных потоков попрежнему значимы. Фактор неопределенности, заданный в данной регрессии как волатильность цен базовых активов, ожидаемо оказался значимым, так как было показано, что волатильность цен производных финансовых инструментов определяется именно этим фактором. Значимость фактора волатильности цен базовых активов в квадрате упала по отношению к аналогичному показателю для производных финансовых инструментов, следовательно, данный фактор не значим на 10\%-ном уровне и им можно пренебречь.

Показатели денежных потоков имеют примерно такую же значимость, как и в первоначальной регрессии.

Итоговая модель выглядит следующим образом:

$I N V=-1,542518+0,085425^{*} I N V_{-} L A G 1-0,042572^{*} U N C_{-} B A+0,002154 * S L S+$ $0,001842 * C F++0,035182 * C F \_L A G 1$,

Данная аналитика показывает, что регрессия значима уже на $1 \%$-ном уровне значимости $(p$-value $=0,000)$. Автокорреляция $(D W$ stat $=1,54)$ и гетероскедастичность в модели отсутствуют. В пакете Stata строилась дополнительная модель, очищенная от гетероскедастичности, которая показывает такие же коэффициенты. Можно сделать вывод об отсутствии мультиколлинеарности, так как корреляция показателей не превышает $24 \%$. 


\section{Исследование периодичности колебаний неопределенности}

Для того чтобы сформулировать советы менеджменту компаний, необходимо построить прогнозную модель колебаний неопределенности.

Для анализа колебаний была исследована следующая зависимость:

$$
\frac{U N C}{U N C_{-} B A}=f(t)
$$

где:

$U N C$ - неопределенность, измеряемая как волатильность цен производных финансовых активов;

$U N C \_B A-$ неопределенность, измеряемая как волатильность цен базовых активов;

$t$ - время в рабочих днях.

Таким образом, изучение данной зависимости призвано продемонстрировать частоту увеличения неопределенности, определяемой как увеличение разрыва между волатильностью цен производных финансовых инструментов и волатильностью цен базовых активов.

Исследование данной модели проводилось с помощью специализированного программного пакета компьютерной алгебры Mathcad, разработанного профессором массачусетского технологического института А. Раздовым специально для анализа и построения сложных функций. Сотни операторов и встроенных функций программы позволяют решать самые различные задачи, например построение полиномиальных регрессий, которые стандартные пакеты не поддерживают.

Система позволяет осуществлять подбор регрессии по параметрам. Полученная регрессия описывается следующим уравнением:

$Y(x)=-\left(6,1709564 * 10^{-23}\right) * x^{9}+\left(2,3658286^{*} 10^{-19}\right) * x^{8}-\left(3,7700583 * 10^{-16}\right) * x^{7}+$

$\left(3,2404619 * 10^{-13}\right) * x^{6}-\left(1,6295925 * 10^{-10}\right) * x^{5}+\left(4,8574765 * 10^{-8}\right) * x^{4}-$

$-\left(8,2672413 * 10^{-6}\right) * x^{3}+\left(7,2449409 * 10^{-4}\right) * x^{2}-0,0254137 * x+1,1891931$

где $Y(x)=U N C / U N C \_B A, x=t$;

$U N C$ - волатильность цен срочных инструментов;

$U N C \_B A-$ волатильность цен базового актива.

Данная функция имитирует изменение аргумента во времени, что позволяет определить периоды увеличения и снижения неопределенности методами имитационного моделирования.

По виду регрессии достаточно сложно что-либо оценить без дополнительных расчетов и преобразований, поэтому целесообразно применить графический анализ построенной модели.

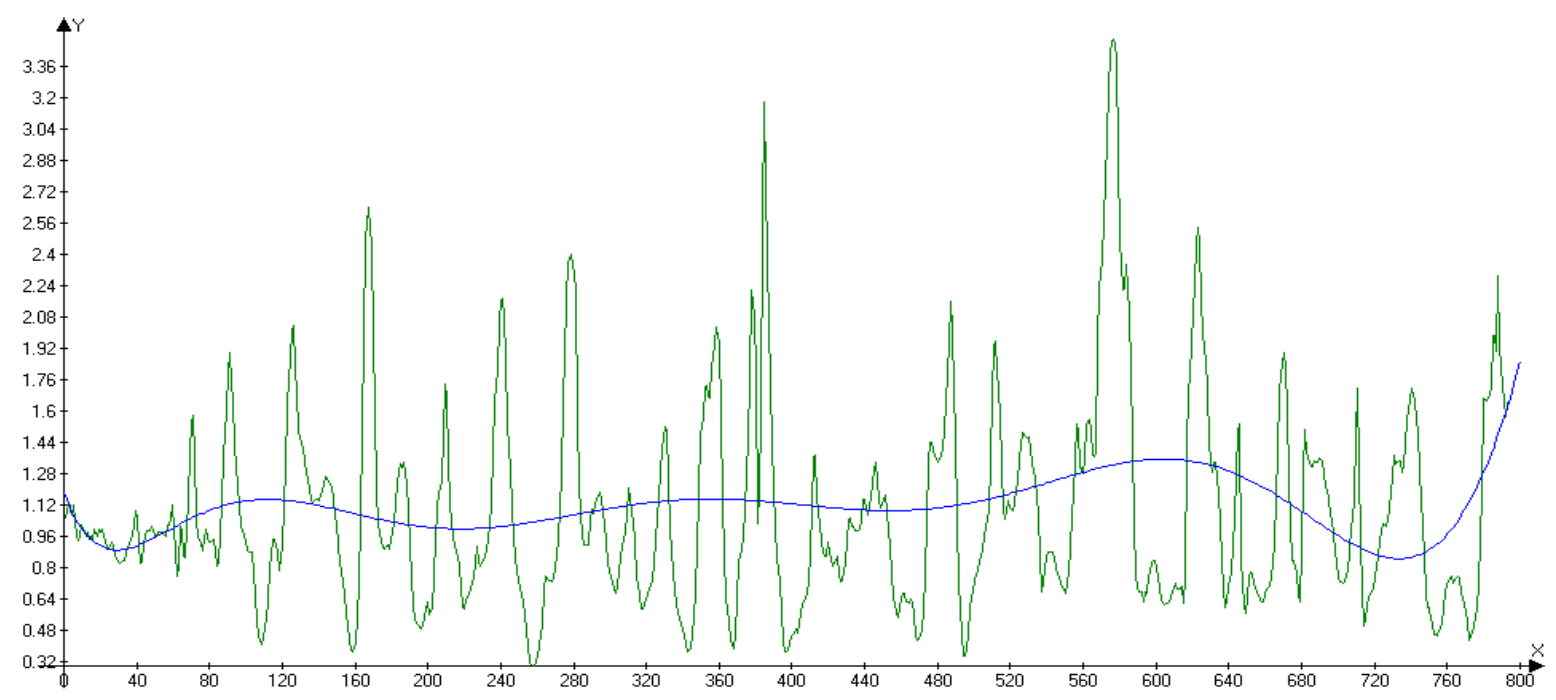

Рисунок 1. Циклические колебания показателя неопределенности во времени 
На приведенном рисунке явным образом прослеживаются колебания, вызванные увеличением и снижением разброса в стандартных отклонениях цен производных финансовых инструментов и базовых активов. По результатам анализа полученной модели были выявлены следующие экстремумы:

Таблица 1

Экстремумы полиномиальной регрессии (расчеты авторов)

\begin{tabular}{|l|l|l|l|}
\hline \multicolumn{1}{|c|}{ Экстремум } & \multicolumn{1}{c|}{ Рабочне дни } & \multicolumn{1}{c|}{ UNC/UNC_BA } & \multicolumn{1}{c|}{ Интервал, дней } \\
\hline Min & 29 & 0.89 & 29 \\
\hline Max & 112 & 1.15 & 83 \\
\hline Min & 218 & 1 & 106 \\
Max & 355 & 1.15 & 137 \\
\hline Min & 454 & 1.09 & 99 \\
Max & 604 & 1.36 & 150 \\
Min & 733 & 0.85 & 129 \\
\hline
\end{tabular}

Интервалы между максимальными значениями составили 243 и 249 рабочих дней соответственно. Средний интервал между пиковыми значениями спреда составляет 246 рабочих дней. Максимальные значения спреда представляют собой периоды, наименее благоприятные для осуществления инвестиций компаний в силу неоднозначности оценки инвестиций в такие периоды. Средний интервал в 123 рабочих дня (примерно 5,5 месяца) представляет собой рекомендуемый для компаний срок отсрочки принятия инвестиционных решений относительно вложений в деятельность, связанную с производством базового актива. Так как одним из основных свойств инвестиций является их невозвратность, то отсрочка принятия инвестиционных решений может повысить стоимость компании за счет того, что удастся избежать неудачных вложений.

Приведенный анализ был проведен на примере цен на нефть марки Brent, а также на примере цен производных инструментов на данную марку. Аналогичные исследования были проведены и для других базовых активов, таких как золото, серебро, палладий и медь.

Для компаний, базовыми активами которых являются металлы, рекомендуются следующие интервалы отсрочки принятия инвестиционных решений:

Таблица 2

Средний интервал отсрочки принятия инвестиционных решений в разрезе базовых активов (расчеты авторов)

\begin{tabular}{|c|c|}
\hline Базовьпй актив компании & $\begin{array}{c}\text { Р екомендуемьй иптерв ап отсрочки принятия } \\
\text { инвестиционньх решений, рабочпх дней }\end{array}$ \\
\hline 3опото & 171 \\
Серебро & 153 \\
Медь & 114 \\
Папладий & 143 \\
\hline
\end{tabular}

\section{Основные выводы}

На основании проведенного исследования можно сделать следующие выводы.

Была подтверждена гипотеза об отрицательном влиянии неопределенности, вызванной колебаниями цен на производные финансовые инструменты, на инвестиционную активность российских компаний. Данный вывод согласуется с многочисленными исследованиями других видов неопределенности (Abdul-Haque, Shaoping, 2008; Abel, Eberly, 1999), в которых авторы неизменно получали отрицательную зависимость данного фактора.

Несмотря на то что по результатам исследования не было получено однозначно выявленной значимости квадратичного показателя неопределенности, нельзя считать подтвержденной гипотезу о наличии квадратичной зависимости инвестиций от 
неопределенности и, соответственно, нельзя сказать, что зависимость имеет U-образную форму. Данный вывод расходится с полученным Садорски и Энрикесом результатом (Henriques, Sadorsky, 2011). Возможно, это объясняется тем, что в их работе была использована достаточно смелая интерпретация результатов, в то время как квадратичная зависимость может иметь место только на протяжении одной ветви параболы.

Что касается других переменных, то параметр лагового влияния выручки, который в некоторых работах значимо и положительно влияет на инвестиции, в данном исследовании признан незначимым. Можно предположить, что данный результат получен в силу того, что компании планируют инвестиции исходя из прогнозов выручки и потоков на период, в котором и будут осуществляться инвестиции. При этом в лаговый показатель выручки может входить множество иных факторов.

Факторы, характеризующие денежный поток, оказались значимыми, что согласуется с теоретическими выкладками и большинством исследований данной проблемы. В то же время лаговый фактор инвестиций также оказался значимым, что явно соответствует теории инвестиционного акселератора и подстройки капитала под оптимальное значение.

Кроме того, для неопределенности, вызванной волатильностью стоимости производных финансовых инструментов, была получена зависимость, демонстрирующая влияние волатильности цен базовых активов и времени, оставшегося до исполнения контракта. При подставлении данной оценки в основную регрессию фактор времени оказался незначимым, тогда как фактор волатильности цен базовых активов - значимым, в отсутствие фактора волатильности цен срочных инструментов.

При моделировании зависимости неопределенности от времени были сымитированы данные о периодичности колебаний волатильности. Также были рассчитаны средние периоды отсрочки принятия инвестиционных решений, рекомендованные для компаний разных отраслей в разрезе базовых активов. Отсрочка инвестиций может позволить компаниям избежать существенных издержек, так как инвестиции обладают свойством невозвратности, вследствие чего при неудачном ходе проекта вложенные средства могут быть потеряны.

\section{Список литературы}

1. Берндт Э.Р. Практика эконометрики: классика и современность: учебник для студентов вузов / пер. с англ. под ред. проф. С.А. Айвазяна. М., 2005.

2. Сошникова Л.А. Статистический анализ инвестиций природоохранного значения // Вопросы статистики. 2009. № 5. С. 43-52.

3. Черкасова В.А. Реализация стратегии в условиях неопределенности.// Проблемы теории и практики управления. 2009. № 2. С. 75-82.

4. Черкасова В.А., Теплова О.Ю. Исследование факторов, влияющих на инвестиционную активность компаний // Корпоративные финансы. 2011. № 3. (19).

5. Abdul-Haque, Shaoping, W. (2008), Uncertainty and investment evidence from a panel of Chinese firms, Structural Change and Economic Dynamics, 19 (2008) 237-248.

6. Abel, A.B., Eberly, J.C. (1997), An exact solution for the investment and value of a firm facing uncertainty, adjustment costs, and irreversibility, Journal of Economic Dynamics and Control, 21 (1997) 831-852.

7. Abel, A.B., Eberly, J.C. (1998), The mix and scale of factors with irreversibility and fixed costs of investment, Carnegie-Rochester Conference Series on Public Policy, 48 (1998) 101-135.

8. Abel, A.B., Eberly, J.C. (1999), A unified model of investment under uncertainty, American Economic Review, 84 (1999) 1369-1384.

9. Barnett, S.A., Sakellaris, P. (1998), Nonlinear response of firm investment to Q: Testing a model of convex and non-convex adjustment costs, Journal of Monetary Economics, 42 (1998) 261-288. 
10. Barro, R.J. (1990), The Stock Market and Investment, Review of Financial Studies, 3 (1990) 47-59.

11. Bloom, N., Bond, S.R., Van Reenen, J. (2003), Uncertainty and Company Investment Dynamics: Empirical Evidence for UK Firms, CEPR, Discussion Papers, 4025.

12. Bond, S., Elston, J., Mairesse, J., Mulkay, B. (1997), Financial factors and investment in Belgium, France, Germany and the UK: acomparison using company panel data, NBER, Working paper.

13. Bond, S., Van Reenen, J. (2003), Microeconometric Models of Investment and Employment. Institute for fiscal Studies (IFS), Handbook of Econometrics, V. 6A.

14. Bonnisseau, J.-M., Lachiri, O. (2004), On the objective of firms under uncertainty with stock markets, Journal of Mathematical Economics, 40 (2004) 493-513.

15. Chirinko, R. S., Schaller, H. (1996), Bubbles, fundamentals, and investment: A multiple equation testing strategy, Journal of Monetary Economics, 38 (1996) 125-144.

16. Drakos, K., Goulas, E. (2006), Investment and conditional uncertainty: The role of market power, irreversibility, and returns-to-scale, Economic Letters, 93 (2006) 169-175.

17. Dzielinski, M. (2011), Measuring economic uncertainty and its impact on the stock market, Finance Research Letters, Article in Press.

18. Eberly, J.C. (1997), International evidence on investment and Fundamentals, European Economic Review, 41 (1997) 1055-1078.

19. Haruna, S. (1996), Industry equilibrium, uncertainty, and futures markets, International Journal of Industrial Organization, 14 (1996) 53-70.

20. Henriques, I., Sadorsky, P. (2011), The effect of oil price volatility on strategic investment, Energy Economics, 33 (2011) 79-87.

21. Kogan, L. (2001), An equilibrium model of irreversible investment, Journal of Financial Economics, 62 (2001) 201-245.

22. Leahy, J., Whited, T. (1996), The effect of uncertainty on investment: Some stylized factors, Journal of Money, Credit and Banking, 37 (1996) 64-83.

23. Moon, Y. (2010), Efforts and efficiency in partial outsourcing and investment timing strategy under market uncertainty, Computers \& Industrial Engineering, 59 (2010) 24-33.

24. Pillania, R.K. (2011), The state of research on technological uncertainties, social uncertainties and emerging markets: A multidisciplinary literature review, Technological Forecasting \& Social Change, 78 (2011) 1158-1163.

25. Slovik, P. (2010), Market uncertainty and market instability, IFC Bulletin 34.

26. Tvedt, J. (2002), The effect of uncertainty and aggregate investments on crude oil price dynamics, Energy Economics, 24 (2002) 615-628. 